\title{
Moral als Kompetenz
}

\section{Agnieszka Vojta (Konstanz)}

Georg Lind, visit this Moral ist lehrbar. Handbuch zur Theorie und Praxis moralischer und demokratischer Bildung, Verlag Oldenbourg, München 2009, SS. 169.

Wie wir von Sokrates gelernt haben, ist Moral eine Tugend, moderner ausgedrückt, eine Fähigkeit. Und somit kann man sie erlernen. Georg Lind plädiert in seinem Grundlagenbuch ?Moral ist lehrbar? für eine frühzeitige Erziehung zur Moral, wobei er davon ausgeht, dass Bildung Moral fördert. Die Förderung moralischer Fähigkeiten soll als integrativer Teil der Ausbildung implementiert und somit allen zugänglich gemacht werden.

Linds Ansatz ist ein Plädoyer für Chancengleichheit ? wenn Moral lernbar ist, nicht genetisch bedingt oder schichtabhängig, kann sie jeder erlernen. Durch die breite Förderung moralischer Kompetenzen Einzelner werden auch die demokratischen Kompetenzen der Gemeinschaften gestärkt.

Das Buch ist in drei Teile gegliedert: Im ersten, theoretischen Teil beschäftigt sich der Autor mit dem Zusammenhang zwischen Moral, Demokratie und Bildung. Im zweiten Teil, dem Kern des Buches, wir die Methode der Dilemmadiskussion detailliert vorgestellt. Im dritten Teil sind Anregungen und Empfehlungen für den (Hoch-)Schulunterricht zusammengetragen.

Demokratie ist eine moralische Institution und umgekehrt ist die moderne Moral demokratisch. Die moderne Moral ist eine individuelle, autonome Moral, sie ist sowohl lern- als auch lehrbar. Die zentrale Aufgabe einer Demokratie ist die Bildung, und zwar nicht nur die Vermittlung von Fachwissen und Kompetenzen, sondern auch die Förderung von moralischer Urteils- und Diskursfähigkeit. Außer moralischen Idealen braucht es auch die Fähigkeit, diese auch umzusetzen bzw. nach ihnen zu handeln. Somit geht es um die Vermittlung moralischer Diskurs- und Urteilsfähigkeit, nicht um reine Wertevermittlung. Die moralische Urteilsfähigkeit wird von Kohlberg definiert als ?die Fähigkeit, das eigene Denken an moralischen Idealen oder Prinzipien auszurichten und auf der Grundlage dieses Denkens zu handeln? (74).

Als erprobte, nachhaltige und praktikable Methode hat sich die Methode der Dilemma Diskussion (Blatt, Kohlberg), bzw. die von Lind weiterentwickelte und evaluierte Konstanzer Methode der Dilemmadiskussion (KMDD) erwiesen.

Durch die detailliert strukturierte Methode der KMDD können auch Grundschulkinder an den Diskussionen teilnehmen und so ihre moralischen Fähigkeiten entwickeln. Entscheidend sind der gegenseitige Respekt und die Achtung, also Prinzipien demokratischer Gemeinschaften. Im Zentrum steht die Auseinandersetzung mit Gegenargumenten, wobei sich Phasen der Unterstützung und der Herausforderung abwechseln, um die Aufmerksamkeit der Teilnehmer zu halten, sie weder zu übernoch zu unterfordern. Im Fokus stehen semi-reale Dilemmas mit einem hohen Realitätsgehalt. Somit konzentrieren sich die Diskutanten auf die Sache statt auf anderen Personen. Den zentralen Teil der KMDD bildet die selbstgesteuerte Diskussion im Plenum, bei der die gegnerischen Gruppen ihre Argumente austauschen. Die Ping-Pong-Regel besagt, dass derjenige die Moderation übernimmt bzw. den nächsten Beitrag wählt, der gerade selber gesprochen hat. Somit lernt die Gruppe ohne eine übergeordnete Autorität zu handeln.

Die KMDD fördert verschiedene Teilfähigkeiten der moralischen Urteils- und Diskurskompetenz, so dass die TeilnehmerInnen imstande sind, den Argumenten anderer zuzuhören, die eigenen Prinzipien in einem sozialen Kontext zu artikulieren, die eigenen Prinzipien nach ihrer Wichtigkeit und Angemessenheit einordnen zu können, Respekt und Toleranz gegenüber anders denkenden zu zeigen. Besonders wichtig erscheint mir die Fähigkeit, ?den Gegner als Instanz zur Überprüfung eigener moralischer Standpunkte schätzen zu lernen? (75).

Georg Lind fordert, dass moralisch-demokratische Kompetenzen auf allen Stufen des schulischen Lernens eingeführt werden und zwar als Bestandteil jedes Fachs. Wichtig ist dabei, die moralisch-demokratische Bildung als Aktivität unter Gleichen anzusehen, und die gewohnte Kluft zwischen Lehrern und Lernenden zu überbrücken.

Um die moralischen Fähigkeiten bzw. ihren Zuwachs überhaupt messen zu können, hat Professor Lind mit seiner Forschergruppe an der Universität Konstanz den ?Moralischen Urteil-Test? (MUT) entwickelt. Der Test besteht aus einem Fragebogen, in dem die Versuchspersonen gebeten werden, die Argumente in zwei moralischen Konfliktsituationen (aktive Sterbehilfe, Datendiebstahl) nach ihrer moralischen Qualität zu beurteilen, unabhängig davon, ob sie die eigene Meinung unterstützen oder ihr widersprechen. Um einen Wert zu bekommen, wird das Urteilsmuster der Versuchsperson strukturell analysiert.

Besonders interessant scheint der Zusammenhang zwischen Bildung und moralischen Kompetenzen: Bildung ist ein zentraler Förderungsfaktor der Moral. 
Es braucht ein bestimmtes Niveau an Allgemeinbildung, um die Selbstlernprozesse zu aktivieren, welche die moralische Entwicklung unterstützen, auch wenn die institutionellen Bildungsprozesse wegfallen. Linds Untersuchungen haben belegt, dass moralische Kompetenzen auch abnehmen können ? die Regression findet eben dann statt, wenn ein bestimmtes Niveau an Allgemeinbildung nicht erreicht werden konnte und die Fähigkeiten nicht so weit entwickelt wurden, dass die Selbstlernprozesse sie unterstützen könnten. Förderlich für die Entwicklung der moralischen Kompetenzen im Unterricht sind weniger die Fachinhalte, als vielmehr die Möglichkeit, Verantwortung zu übernehmen, Freiräume und vor allem angeleitete Reflexion und Beratung.

Im letzten Teil des Buches findet man Unterrichtsmaterialien, Ablaufpläne und zahlreiche Beispiele für Unterrichtsdilemmas. Professor Georg Lind bietet Kurse und Weiterbildungsseminare an. Alle aktuellen Informationen werden auf seiner Homepage veröffentlicht: www. uni-konstanz.de/ag-moral.

Das vorliegende Buch ist einerseits die Auseinandersetzung mit Moralphilosophie und bildet das bildungstheoretische Fundament der Förderung von moralisch-demokratischer Kompetenzen, gleichzeitig ist es ein anschauliches und praxisnahes Handbuch zur Einführung der KMDD im Unterricht. Wichtig scheint die Erkenntnis, dass Förderung moralischer Kompetenzen keinesfalls auf Ethikunterricht beschränkt sein sollte, sondern in allen Fächern einsetzbar ist.

Fazit: Moral ist lehrbar und lernbar und sie zu lehren bzw. zu lernen kann sogar Spaß machen, wenn man die Konstanzer Methode der Dilemma Diskussion anwendet. 